\title{
ON THE MAGNETIC STRUCTURE OF AN ACTIVE REGION*
}

\author{
F. A. Ermakov, E.I. Mogilevsky, and B.D. Shelting \\ (Institute of Terrestrial Magnetism, Ionosphere and Radio-Wave Propagation, \\ Academy of Sciences, Moscow, U.S.S.R.)
}

\begin{abstract}
A BSTRACT
(1) A scheme of magnetic-field generation in an active region within the subphotospheric convective zone is discussed. Magnetic-field amplification is considered to result from the interaction of the local cyclonic motion and the turbulent motion of the subphotospheric plasma.

(2) A model of the magnetoplasma of an active region is considered consisting of a current-free magnetic field, whose lines of force stretch deeply under the photosphere in the form of subgranules (force-free fine-scale plasma elements). The explanation of some peculiarities in the development of an active region with the considered structure is discussed.

(3) Some peculiarities of the general structure of the magnetic field and the motion within photosphere and chromosphere obtained by the analysis of observations with the IZMIRAN two-channel magnetograph is discussed.
\end{abstract}

\section{DISCUSSION}

H.U. Schmidt: What size do you have in mind for the subgranules? Why did you choose this size? Mogilevsky: The calculated size of the subgranules is $\sim 10^{7} \mathrm{~cm}$. This estimation of the high limit of the subgranule middle size was obtained from the equation of the integral magnetic flux over the whole active region by present scheme 'magnetisation' of the magnetoplasma.

* A detailed paper will be submitted to Solar Physics.

Kiepenheuer (ed.), Structure and Development of Solar Active Regions, 126. ‘. I.A.U. 